\title{
Preparation and Experimental Study of Magnetorheological Fluids for Vibration Control
}

\author{
Ying-Qing Guo \\ Mechanical and Electronic Engineering College, Nanjing Forestry University, Nanjing 210037, China
}

Zhao-Dong Xu and Bing-Bing Chen

Key Laboratory of C\&PC Structures of the Ministry of Education, Southeast University, Nanjing, 210096, China

\author{
Cheng-Song Ran
}

Sichuan Provincial Architectural Design and Research Institute, Chengdu 610017, China

\author{
Wei-Yang Guo \\ Nanjing Dongrui Damping Control Technology Co, Ltd, Nanjing 210033, China
}

(Received 1 May 2015; accepted 19 April 2016)

\begin{abstract}
Magnetorheological (MR) fluids are a new kind of smart vibration mitigation material for vibration control, whose shear yield stress can change in magnetic field, and the change can occur only in a few milliseconds. The rheological properties, anti-settlement stability, and redispersibility are the very important properties of MR fluids. In this paper, a kind of preparation process of MR fluids is introduced and MR fluids with different grain diameter ratios of carbonyl iron particles are produced. Then, the properties of self-prepared MR fluids are tested, including the sedimentation stability test, viscosity test, and shear yield stress test. The results of the MR fluid property tests show that the adding of $10 \mu \mathrm{m}$-carbonyl iron particles will improve the magnetic effect of MR fluids, increase the zero magnetic field viscosity of MR fluids, and increase shear yield stress of MR fluids in same magnetic field, but the anti-settlement properties will be degraded.
\end{abstract}

\section{INTRODUCTION}

MR fluids are a kind of controllable fluid that were identified by the US National Bureau of Standards in 1948. ${ }^{1}$ MR fluids usually consist of micrometer-sized magnetic particles, a dielectric carrier fluid, and some additives. When they are placed in an adjustable magnetic field, their yield stress changes with the magnetic field intensity changes in a few milliseconds. Because of this kind of smart feature of MR fluids is that they have been concerned by more and more researchers. Furthermore, MR fluids can be designed as MR dampers to reduce the different vibration. And MR fluids have been successfully used in intelligent vibration control, such as the building structures, the bridge structures, the automobile suspension system, the prosthetic limb, some military equipment or magnetorheological fishing. A large-scale and 20 ton MR fluid damper has been designed and built to reduce vibration for civil engineering applications. ${ }^{2}$ Carlson $^{3}$ used the MR damper to develop an artificial limb. Lord Corporation ${ }^{4}$ used MR dampers for the vibration control of armored vehicles. Xu et al. ${ }^{5}$ used the MR damper for mitigating earthquake responses of building structure under the neural networks control strategy.

The properties of MR fluids will directly influence the vibration absorption performance of MR dampers, so many researchers have studied how to prepare MR fluids to improve the properties of MR fluids. In 2004, Ulicny and Mance ${ }^{6}$ studied the anti-oxidation property of MR fluids and proposed the method with plating ferromagnetic particles coated in a layer of nickel to improve the long-term oxidation resistance of MR fluids. In 2010, Jiang et al. ${ }^{7}$ coated the poly (methyl methacrylate) (PMMA) to the surface of carbonyl iron (CI) particles getting the composite particle CI-PMMA. The prepared MR fluids based on CI-PMMA particles had better sedimentation stability. In 2010, Du et al. ${ }^{8}$ chose the surfactant by hydrophilic-lipophilic balance (HLB) parameters and discovered that the surfactant could hardly change the magnetic properties of magnetic particles and rheological properties of MR fluids, while increasing the sedimentation stability of MR fluids significantly. In 2012, Iglesias et al. ${ }^{9}$ tested the influence of the volume fraction of nanoscale magnetic particles on the sedimentation stability. Powell et al. ${ }^{10}$ synthesized MR fluids by replacing a part of the magnetic particles with nonmagnetic microscale glass bead, and the sedimentation rate was reduced by about $4 \%$. In 2011 , Lee et al. ${ }^{11}$ applied tribological characteristic to improve the stability and performance of MR fluids by mixing and adding the additives. In 2013 , Liu et al. ${ }^{12}$ proposed a preparation method of silicone oil-based MR fluids and tested the properties of the prepared MR fluids. However, due to the density mismatch between the ferromagnetic particles and the carrier liquids, the particle sedimentation caused by 
gravitational forces came to be a serious drawback in the use of MR fluids. Sutrisno et al. ${ }^{13}$ prepared a high viscosity MR fluids based on grafted poly-iron particles and the fluids have an excellent thermo-oxidative stability with a nearly constant viscosity. Pramudya et al. ${ }^{14}$ developed novel MR fluids using modified silicone oil, composite polyurethane microsphere additives, and surface-coated iron particles; the addition of composite polyurethane microsphere increased the strength of the fluids, providing well dispersion stability. In 2014, SusanResiga et al. ${ }^{15}$ studied the influence of composition on the yield stress and flow behavior of concentrated ferrofluid-based MR fluids and micrometer-sized iron particles to a concentrated ferrofluid without any supplementary stabilizing agent. The results showed that a direct and simple way to control the magnetorheological and magnetoviscous behavior, as well as the saturation magnetization of the resulting nano-micro composite fluid to fulfill the requirements of their use in various MR control and rotating seal devices. Shah et al. ${ }^{16}$ prepared a new kind of low sedimentation MR fluid based on plate-like iron particles and its salient properties were evaluated using a small-sized damper. The results showed that plate-like micron size iron particles played an important role in improving stability against rapid sedimentation as well as in enhancing the value of the yield stress.

In this paper, a kind of preparation process of MR fluids is introduced and MR fluids with different grain diameter ratio of carbonyl iron particles are produced. Then, the properties of self-prepared MR fluids are tested, including sedimentation stability test, viscosity test, and shear yield stress test. The results of property tests of MR fluids show that the adding of $10 \mu \mathrm{m}$-carbonyl iron particles will improve the magnetic effect of MR fluids, increase the zero magnetic field viscosity of MR fluids, and increase the shear yield stress of MR fluids in same magnetic field. However, the anti-settlement properties will be degraded.

\section{PREPARATION OF THE MR FLUID}

\subsection{Materials and instruments}

The materials and instruments for preparation of MR fluids are as follows:

\subsubsection{Materials}

Materials for preparation of MR fluids include $3 \mu \mathrm{m}$ carbonyl iron (CI) particles (produced by Tianyi Co., Ltd. Jiangsu, China; average grain diameter: $3 \mu \mathrm{m}$; density: $7.85 \mathrm{~g} / \mathrm{cm}^{3}$ ), $10 \mu \mathrm{m}$-CI particles (produced by Tianyi Co., Ltd. Jiangsu, China; average grain diameter: $10 \mu \mathrm{m}$; density: $7.85 \mathrm{~g} / \mathrm{cm}^{3}$ ), mineral oil, organobentonite, polyethylene glycol, graphite, polyvinylpyrrolidone, sodium dodecyl benzene sulfonate and oleic acid. Note that all the materials were adopted directly.

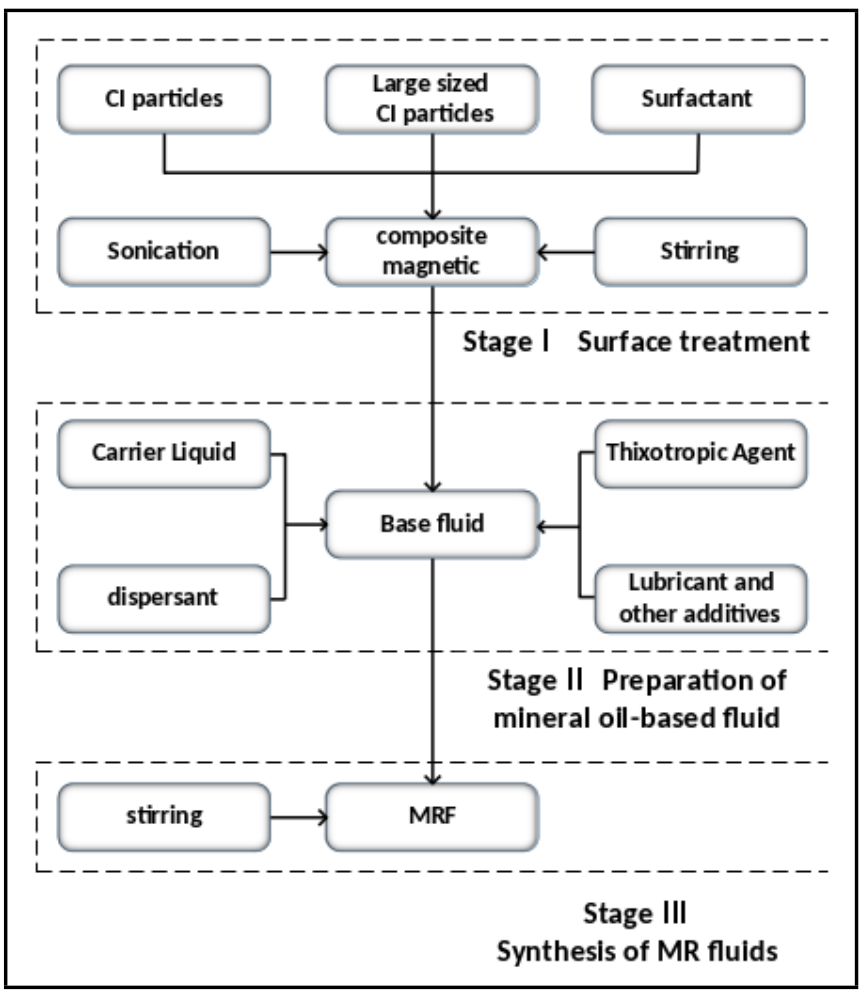

Figure 1. Preparation process of MR fluids.

\subsubsection{Instruments}

The adjustable mixer, the vacuum drying oven, the mortar, the standard sieve (100 mesh), the planetary ball mill, the ultrasonic dispersion instrument and a high precision electronic balance are used to prepare MR fluids.

\subsection{The Preparation Process of the MR Fluid}

The preparation process is an important factor that affects the performance of MR fluids. As shown in Fig. 1, the preparation process can be described in detail as follows:

1. The surface treatment of CI particles: Firstly, put CI particles in a beaker of anhydrous ethanol, then add surfactant. Secondly, put the beaker in the ultrasonic dispersion instrument to make the mixture evenly. Then, place the mixture in vacuum drying oven to keep low temperature drying. Lastly, crush the obtained dry CI particles in a mortar and then sift the particles through a standard sieve (100 mesh).

2. Preparation of mineral oil-based fluid: Take mineral oil into a beaker, add the dispersant, thixotropic agent, and solid lubricant in order while stirring at room temperature for 2 hours.

3. Synthesis of MR fluids: Put the $3 \mu \mathrm{m}$-CI particles and the $10 \mu \mathrm{m}$-CI particles after surface treatment into the obtained mineral oil-based fluid and stir well. According to the above mentioned process of MR fluids, four samples numbered A, B, C and D were prepared. The mass fraction of the total CI particles in each sample was $80 \%$ and 


\begin{tabular}{|c|c|c|c|c|c|}
\hline \multirow{2}{*}{\multicolumn{2}{|c|}{ Materials }} & \multicolumn{4}{|c|}{ The mass percentage } \\
\hline & & \multirow{2}{*}{$\begin{array}{c}\text { Sample A } \\
72.0\end{array}$} & \multirow{2}{*}{$\begin{array}{c}\text { Sample B } \\
74.0\end{array}$} & \multirow{2}{*}{$\begin{array}{c}\text { Sample C } \\
76.0\end{array}$} & \multirow{2}{*}{$\begin{array}{c}\text { Sample D } \\
78.0\end{array}$} \\
\hline CI & $3 \mu \mathrm{m}$ & & & & \\
\hline particles & $10 \mu \mathrm{m}$ & 8.0 & 6.0 & 4.0 & 2.0 \\
\hline \multicolumn{2}{|c|}{$\begin{array}{l}\text { The ratio of } 3-\mu \mathrm{m} \\
\text { CI particles to } \\
10-\mu \mathrm{m} \text { CI particles }\end{array}$} & $9: 1$ & $37: 3$ & 19:1 & 39:1 \\
\hline \multicolumn{2}{|c|}{ Mineral oil } & 16.5 & 16.5 & 16.5 & 16.5 \\
\hline \multicolumn{2}{|c|}{ Thixotropic agent } & 0.6 & 0.6 & 0.6 & 0.6 \\
\hline \multicolumn{2}{|c|}{ Antioxidant } & 0.4 & 0.4 & 0.4 & 0.4 \\
\hline \multicolumn{2}{|c|}{ Solid lubricant } & 0.5 & 0.5 & 0.5 & 0.5 \\
\hline \multicolumn{2}{|c|}{ Dispersing agent } & 1.0 & 1.0 & 1.0 & 1.0 \\
\hline \multicolumn{2}{|c|}{ Surface active agent } & 1.0 & 1.0 & 1.0 & 1.0 \\
\hline
\end{tabular}

the ratio of the $3 \mu \mathrm{m}$-CI particles to the $10 \mu \mathrm{m}$-CI particles are 9:1,37:3, 19:1 and 39:1, respectively. That is, the $10 \mu \mathrm{m}$-CI particles in the proportion of the total CI particles is $10 \%, 7.5 \%, 5.0 \%$ and $2.5 \%$, respectively. As shown in Table 1, the type and amount of other additives were exactly the same.

\section{PROPERTY TEST ON MR FLUIDS}

The sedimentation stability, rheological properties, and high shear yield stress are three main indexes to evaluate the performance of MR fluids. Thus, the prepared four samples of MR fluids in this study were tested to evaluate these properties.

\subsection{Sedimentation stability test}

The sedimentation stability of the MR fluids can be represented by the sedimentation rate, which can be defined as the ratio of the supernatant fluid volume to the mixture volume within a fixed period. The sedimentation ratio is defined as:

$$
\begin{aligned}
& \text { Sedimentation ratio }(\%)= \\
& \quad \frac{\text { volume of the supernatant liquid }}{\text { volume of the entire mixture liquid }} \times 100 \% \text {. }
\end{aligned}
$$

In this paper, the four samples of MR fluids were placed in the four 10-ml graduated glass tubes marked in $0.2-\mathrm{ml}$, respectively, as shown in Fig 2a. The volume of the supernatant liquid was obtained by observing the phase boundary between the supernatant liquid and the concentrated suspension until this volume reached an asymptotic value. Figure $2 b$ shows the sedimentation stability of the MR fluids samples with different amount of $10 \mu \mathrm{m}$-CI particles. It can be seen from the figure that the sedimentation rates of all the samples were relatively large during the first three days and then the rate slowed and tended to be constant after ten days. At the same time, it can be seen that the initial sedimentation rate of the sample $\mathrm{D}$ is much less than that of the sample $\mathrm{C}, \mathrm{B}$, and $\mathrm{A}$. The average final sedimentation rate of the sample A, B, C, and D were $18.2 \%$, $14.9 \%, 14.1 \%$, and $9.8 \%$, respectively. And the average final sedimentation rate of the sample $\mathrm{D}$ was $8.4 \%, 5.1 \%, 4.3 \%$ less than that of the sample A, B, C, respectively. The results show that the sedimentation stability of the MR fluids is relevant to

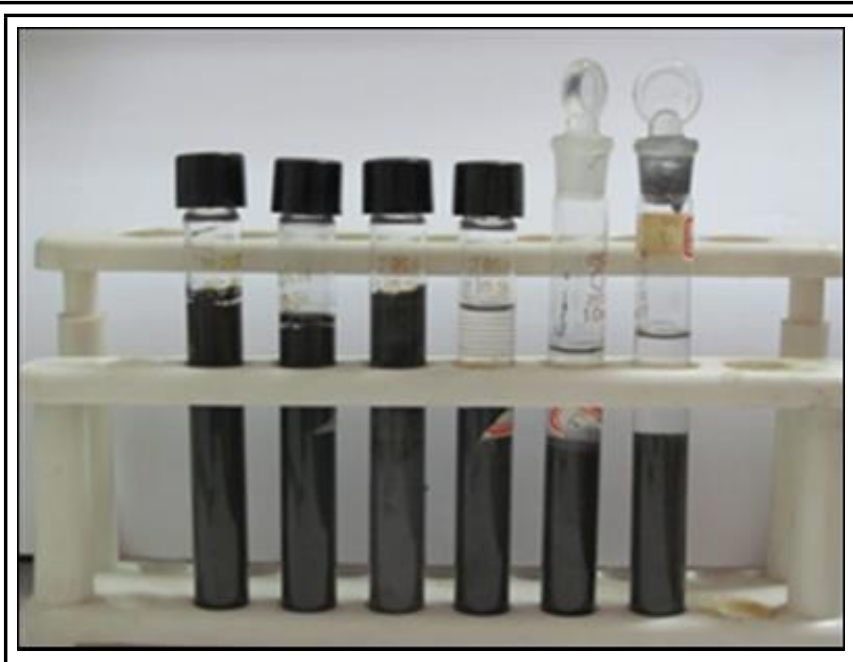

(a) The sedimentation observation.

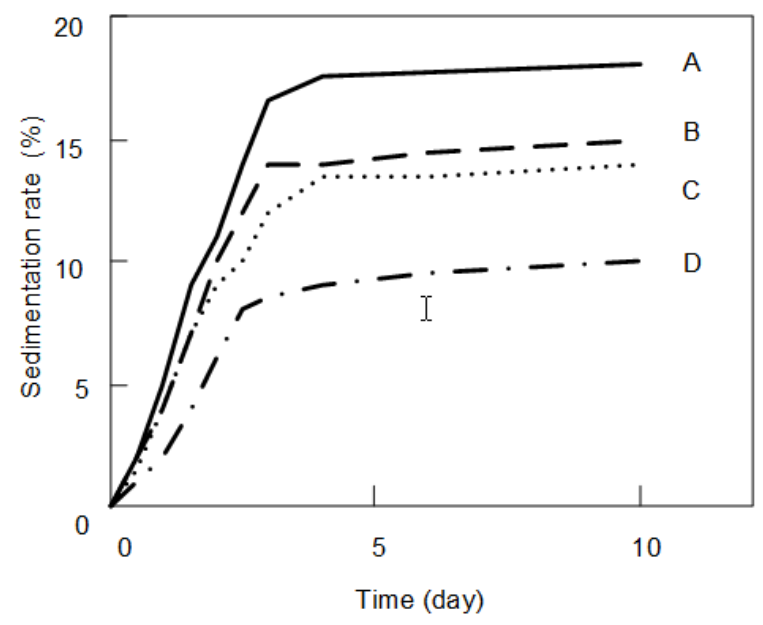

(b) The sedimentation rate.

Figure 2. Sedimentation stability test of MR fluids.

the grain diameter of the CI particles. That is, the larger the ratio of $3 \mu \mathrm{m}$-CI particles, the slower the settlement.

\subsection{Viscosity Test}

In the absence of an external magnetic field, the viscosity of MR fluids mainly demonstrates the flow characteristic of MR fluids under different shear rates, and MR fluids can be thought as Newtonian fluids. An NDJ-1 rotational viscometer produced by Shanghai Jingchun Instrument Equipment Co., Ltd. was adopted to perform the viscosity tests, as shown Fig. 3a. All the samples were carried out the viscosity test under different circular shear rates, $6.28 \mathrm{rad} / \mathrm{s}, 12.57 \mathrm{rad} / \mathrm{s}, 31.42 \mathrm{rad} / \mathrm{s}$, and $62.83 \mathrm{rad} / \mathrm{s}$. The circular shear rate is produced when the rotational viscometer rotates to measure the zero magnetic field viscosity of MR fluids.

Figure $3 b$ shows the viscosity of the samples under the different shear rates with the zero magnetic field. The zero magnetic field viscosities of samples A, B, C, and D are 5.20 Pa.s, 4.74 Pa.s, 4.52 Pa.s, and 4.2 Pa.s, respectively with the shear rate of $6.28 \mathrm{rad} / \mathrm{s}$. It shows that with the increase of the proportion of $10 \mu \mathrm{m}$-CI particles, the zero magnetic field viscosity 


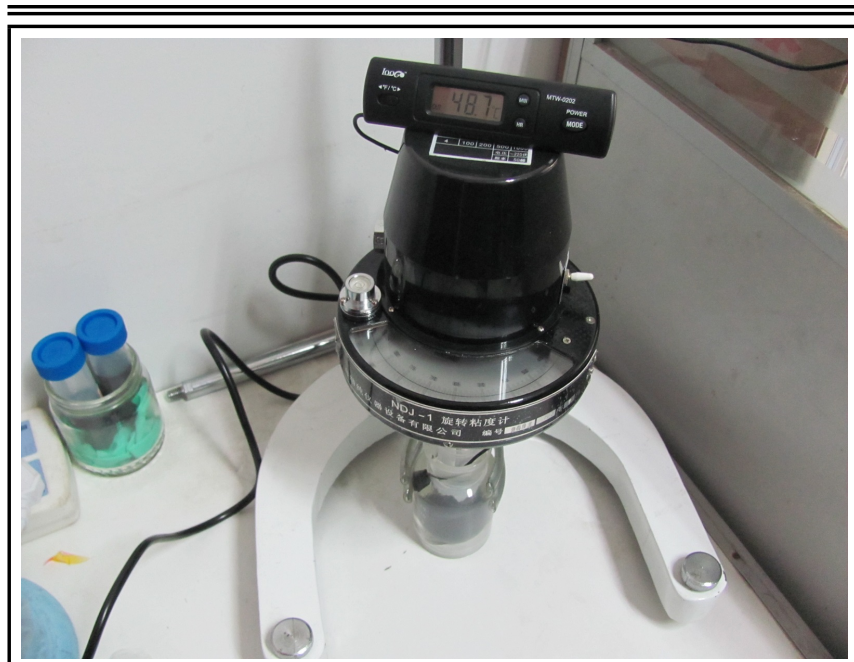

(a) Viscosity test.

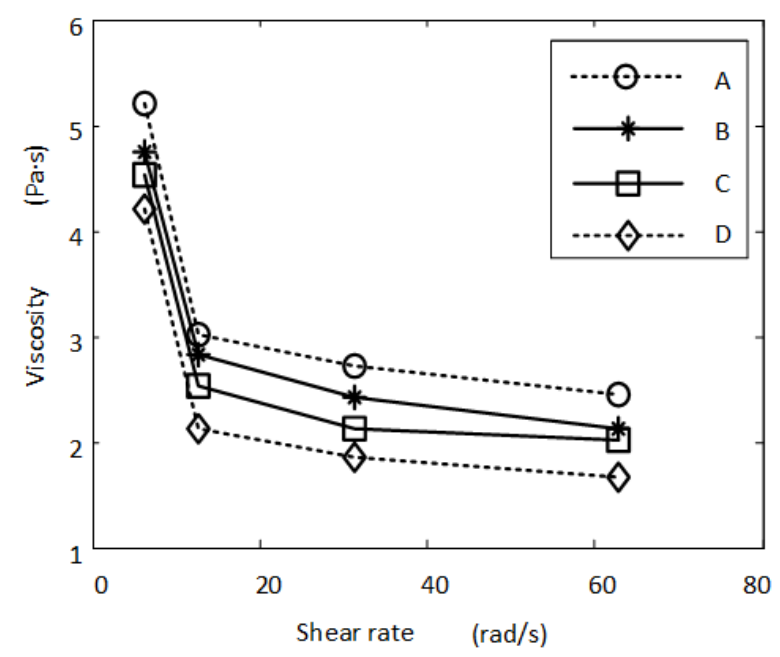

(b) The zero magnetic field viscosity of MR fluids under different shear rates.

Figure 3. Viscosity test of MR fluids under different shear rates.

of MR fluids increases. At the same time, with an increase in the shear rate, the zero magnetic field viscosity of MR fluids decreased gradually. This phenomenon conforms to the theory of shear-thinning.

\subsection{Shear Yield Stress Test}

In an external magnetic field, the CI particles will line up to form a chain-like microstructure, restrict the motion of the fluid, and increase in their ability to support shear stress, as shown in Fig. 4. The shear yield stress of MR fluids will change with the change of the different magnetic fields and shear rates. The shear stress, $\tau$ can be described as Bingham model, ${ }^{17}$

$$
\tau=\tau_{y}(H) \operatorname{sgn}(\dot{\gamma})+\eta \dot{\gamma}
$$

where $\dot{\gamma}$ is the shear rate, $\eta$ is the field-independent plastic viscosity defined as the slope of the measured shear stress versus shear rate, $\tau_{y}$ is the shear yield stress, and $H$ is the magnetic induction intensity. The value of the shear yield stress will embody the damping of the damper with MR fluids.

In this paper, a self-developed shear stress test device (as
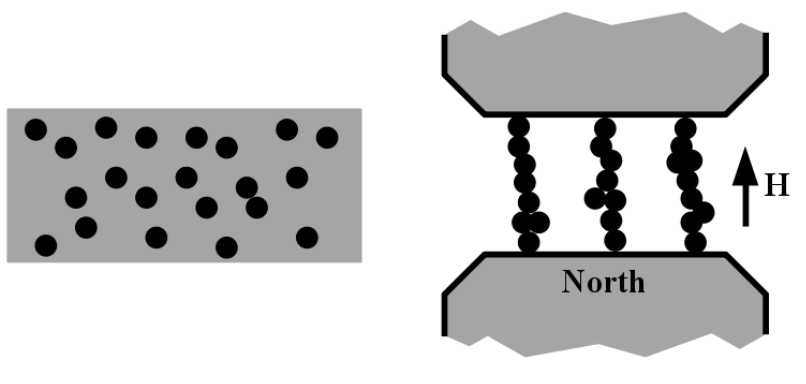

Figure 4. MR fluids.

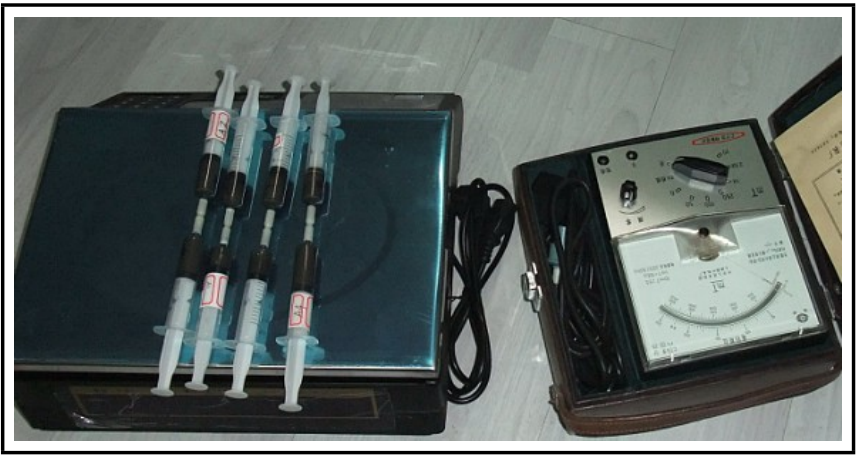

Figure 5. Self-developed shear yield stress test device.

shown in Fig. 5) was used to measure shear yield stress of the sample A, B, C, and D under the different magnetic induction strengths and shear velocities. The shear velocity was produced when the self-developed shear stress device moved rectilinearly to measure the shear yield stress of MR fluids. The shear velocities are: $3.65 \mathrm{~m} / \mathrm{s}, 5.48 \mathrm{~m} / \mathrm{s}, 7.31 \mathrm{~m} / \mathrm{s}, 9.14 \mathrm{~m} / \mathrm{s}$, and $10.97 \mathrm{~m} / \mathrm{s}$. At the same time, the magnetic induction intensity are: $10 \mathrm{mT}, 20 \mathrm{mT}, 25 \mathrm{mT}, 65 \mathrm{mT}$, and $115 \mathrm{mT}$. Figure 6 shows the shear yield stress of the MR fluids samples as a function of external magnetic induction intensity under five different shear velocities, $3.65 \mathrm{~m} / \mathrm{s}, 5.48 \mathrm{~m} / \mathrm{s}, 7.31 \mathrm{~m} / \mathrm{s}$, $9.14 \mathrm{~m} / \mathrm{s}$, and $10.97 \mathrm{~m} / \mathrm{s}$, respectively. It can be seen from Fig. 6 that the shear yield stress of the MR fluid samples A, B, $\mathrm{C}$, and $\mathrm{D}$ increase with an improvement in magnetic induction intensity. Additionally, the shear yield stress value of sample A increases to $80 \mathrm{kPa}$ when the magnetic induction intensity is $115 \mathrm{mT}$, which shows that the MR fluids has a high shear yield stress. Meanwhile, the MR fluids exhibit a typical monotonic improvement in shear yield stress with an increase of magnetic induction intensity as well as Bingham plastic behavior characterized by a field dependent yield stress. At the same time, it also can be seen that the shear yield stress of sample A and D is respectively the biggest and minimum value when magnetic induction intensity and shear rate are given. It demonstrates that the shear yield stress of the MR fluids improve with the increase of the amount of $10 \mu \mathrm{m}$-CI particles.

Figure 7 shows the change in shear yield stress of the MR fluids samples as a function of shear rate under five different magnetic induction intensity, $10 \mathrm{mT}, 20 \mathrm{mT}, 25 \mathrm{mT}, 65 \mathrm{mT}$, and $115 \mathrm{mT}$, respectively. The shear yield stress under the 


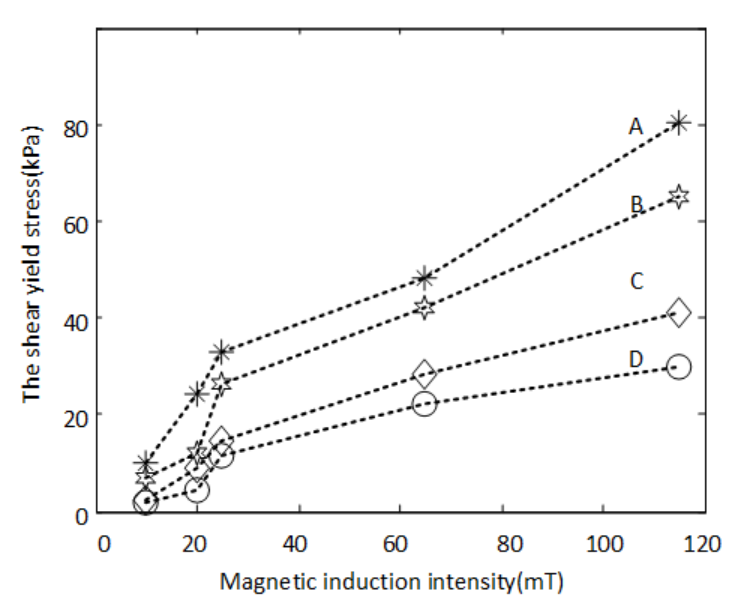

(a) $3.65 \mathrm{~m} / \mathrm{s}$

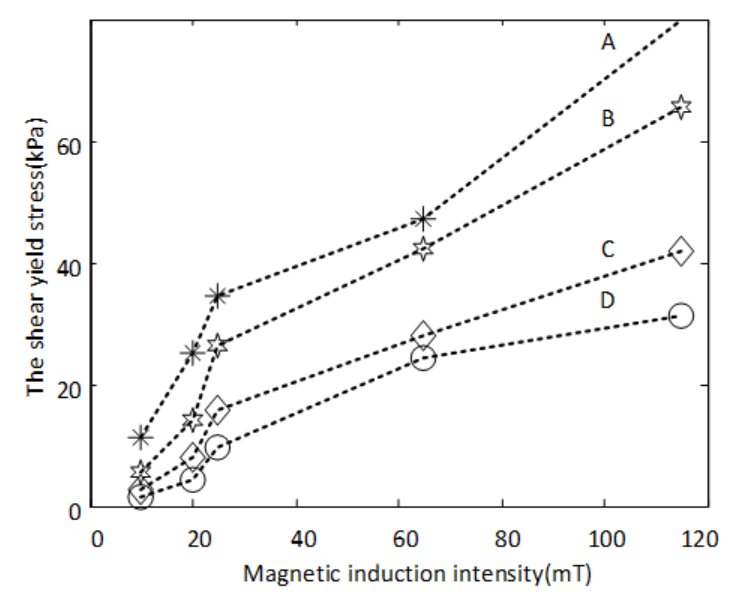

(c) $7.31 \mathrm{~m} / \mathrm{s}$

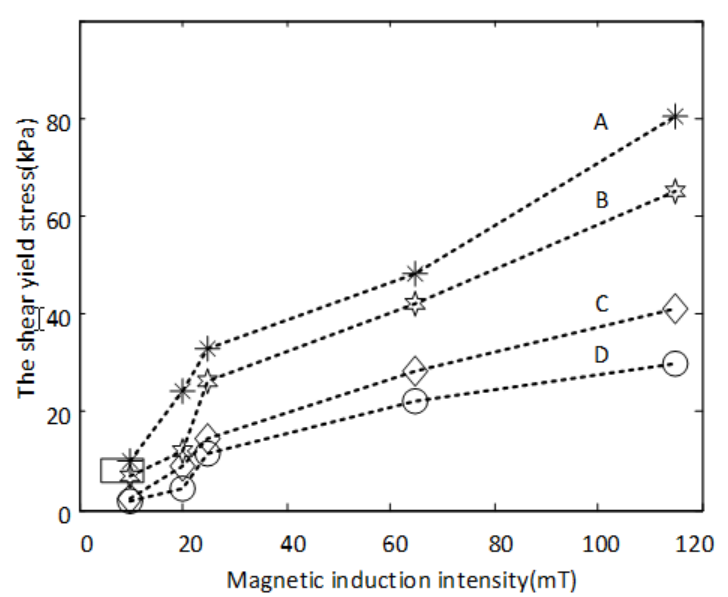

(b) $5.48 \mathrm{~m} / \mathrm{s}$

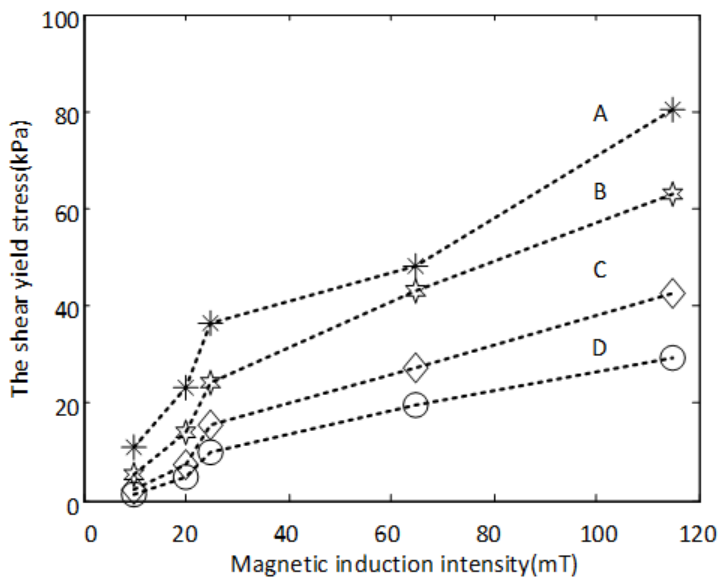

(d) $9.14 \mathrm{~m} / \mathrm{s}$

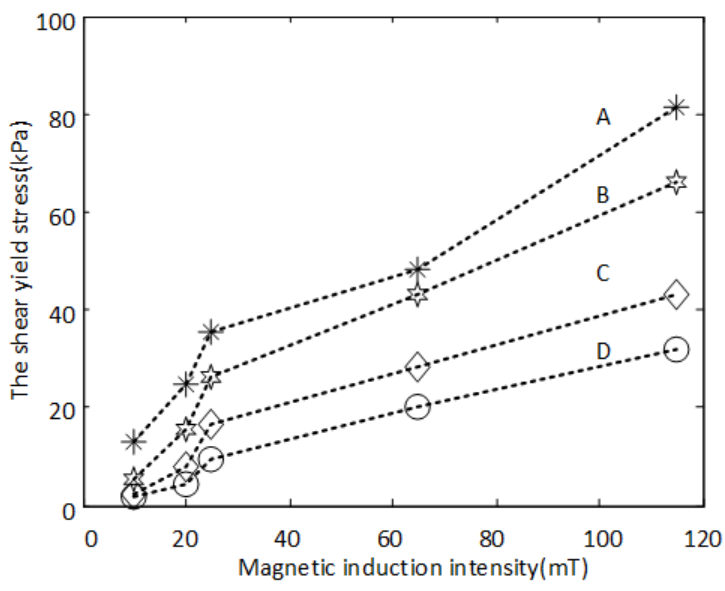

(e) $10.97 \mathrm{~m} / \mathrm{s}$

Figure 6. Shear yield stress under different magnetic fields and shear rates.

magnetic induction intensity ranging from $10 \mathrm{mT}$ to $115 \mathrm{mT}$ fluctuates at a limited amplitude with an increase of shear velocity, which indicates that the shear velocity has little impact on the shear yield stress. The result implies that the chain structure of the MR fluids constructed within fluids under the applied magnetics induction intensity are continuously broken and then rapidly reformed, so that MR fluids are relatively insensitive to shear velocity.

\section{CONCLUSIONS}

In this study, MR fluids were prepared with the appropriate components and process. The $10 \mu \mathrm{m}-\mathrm{CI}$ particles were used to study the effect of the size on the MR fluids performance. To investigate the performance of the MR fluids and determine the effect of $10 \mu \mathrm{m}$-CI particles, some tests, including sedimentation stability, rheological properties, and high shear yield stress were carried out. The following conclusions can be drawn. 


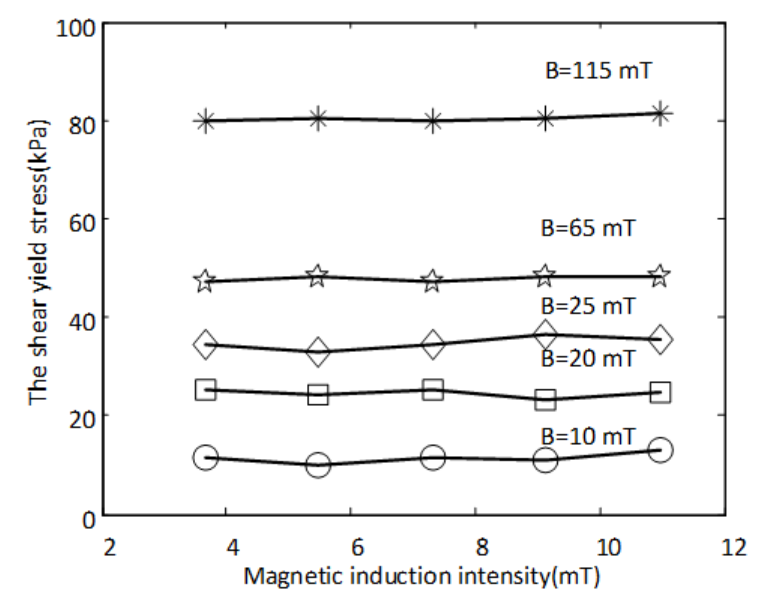

(a) Sample A

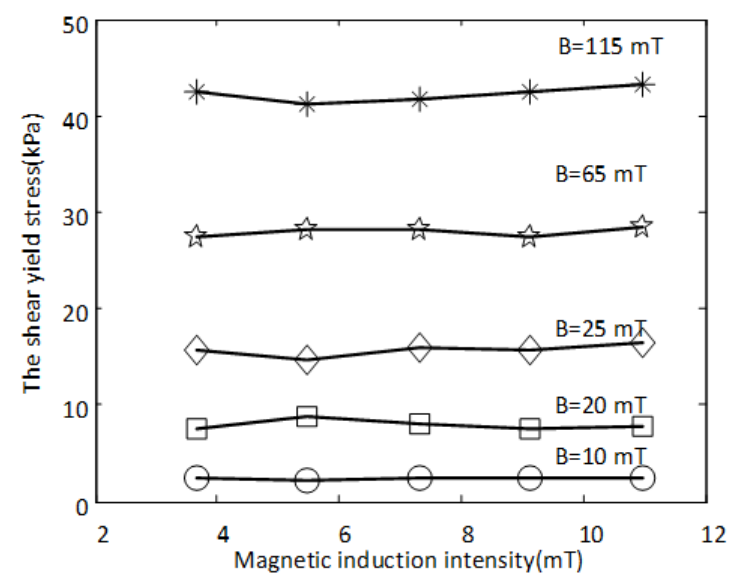

(c) Sample C

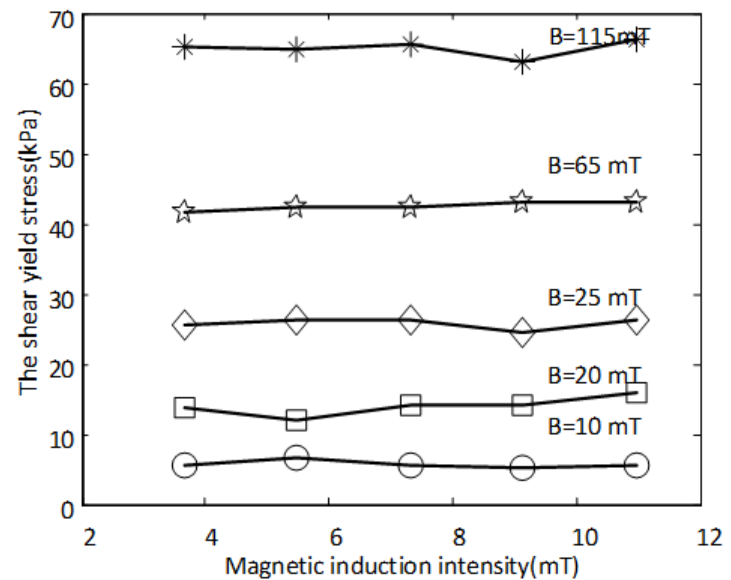

(b) Sample B

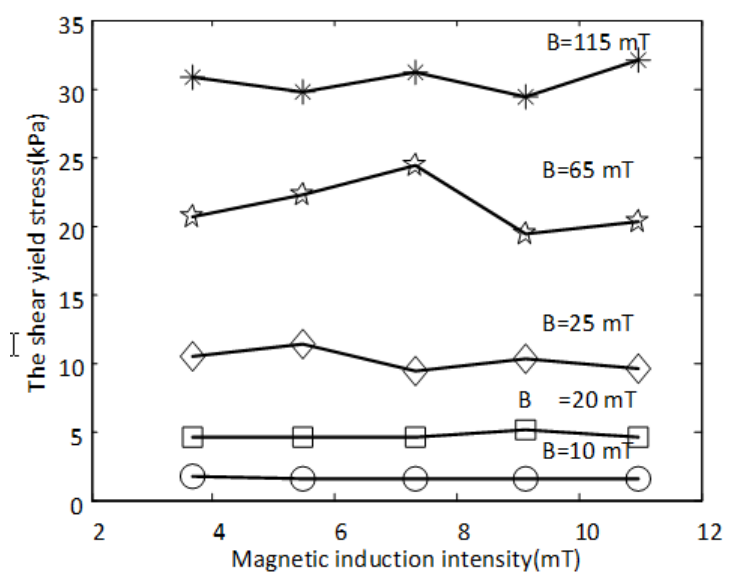

(d) Sample D

Figure 7. Shear yield stress versus shear rates under different magnetic induction intensity.

1. The settlement results show that the sedimentation stability of MR fluids are relevant to the grain diameter of the CI particles. The smaller the particle size, the slower the settlement.

2. The rheological properties tests indicate that the addition of $10 \mu \mathrm{m}$-CI particles can lead to an increase in zero magnetic field viscosity of MR fluids, which shows the grain diameter of CI particles has much effect on rheological properties of MR fluids.

3. The shear yield stress of MR fluids improve with the increase of the amount of $10 \mu \mathrm{m}$-CI particles, which proves that the $10 \mu \mathrm{m}$-CI particles can improve the magnetic effect of MR fluids. At the same time, the shear velocity has little impact on the shear yield stress under the different magnetic induction strengths.

\section{ACKNOWLEDGEMENTS}

This study was financially supported by National Science Fund for Distinguished Young Scholars (51625803),
Jiangsu Province Outstanding Youth Natural Science Foundation (BK20140025), Jiangsu Province Natural Science Foundation (BK20141086), Scientific Research Foundation for Returned Scholars of the Ministry of Education of China, and Jiangsu Province Qing Lan Project. The support is gratefully acknowledged.

\section{REFERENCES}

1 Rabinow, J. The magnetic fluid clutch, Electrical Engineering, 67 (12), 1167-1167, (1948). https://dx.doi.org/10.1109/ee.1948.6444497

2 Yang, G., Spencer, B. F., and Jung H. Dynamic modeling of large-scale magnetorheological damper systems for civil engineering applications, Journal Engineering Mechanics ASCE, 130 (9), 11071114, (2004). https://dx.doi.org/10.1061/(asce)07339399(2004)130:9(1107)

3 Carlson, J. D., Matthis, W., and Toscano, J. R. Smart prosthetics based on magnetorheological fluids, SPIE 8th Annual Symposium on Smart Struc- 
tures and Materials, Newport Beach, CA, (2001). https://dx.doi.org/10.1117/12.429670

4 Lord Corporation. MR suspension technology, http://mutualhosting.com/ lordfulfillment/ upload/PB7138.pdf, (2009).

5 Xu, Z. D., Shen, Y. P., and Guo, Y. Q. Semi-active control of structures incorporated with magnetorheological dampers using neural networks, Smart Materials and Structures, 12 (1), 80-87, (2003). https://dx.doi.org/10.1088/0964$1726 / 12 / 1 / 309$

6 Ulicny, J. C. and Mance, A. M. Evaluation of electroless nickel surface treatment for iron powder used in MR fluids, Materials Science and Engineering: A, 369 (1-2), 309-313, (2004). https://dx.doi.org/10.1016/j.msea.2003.11.039

7 Jiang, W. Q., Zhu, H., Guo, C. Y., Li, J. F., Xue, Q., Feng, J. H., and Gong X. L. Poly (methyl methacrylate)coated carbonyl iron particles and their magnetorheological characteristics, Polymer International, 59 (7), 879-883, (2010). https://dx.doi.org/10.1002/pi.2794

8 Du, C. B., Chen, W. Q., and Wan, F. X. Influence of HLB parameters of surfactants on properties of magneto-rheological fluid, Advanced Materials Research, 97-101, 843-847, (2010). https://dx.doi.org/10.4028/www.scientific.net/amr.97101.843

9 Iglesias, G. R., Lopez-Lopez, M. T., Duran, J. D. G., Gonzalez-Caballero, F., and Delgado, A. V. Dynamic characterization of extremely bidisperse magnetorheological fluids, Journal of Colloid and Interface Science, 377 (1), 153-159, (2012). https://dx.doi.org/10.1016/j.jcis.2012.03.077

10 Powell, L. A., Wereley, N. M., and Ulicny, J. Magnetorheological fluids employing substitution of nonmagnetic for magnetic particles to increase yield stress, IEEE Transactions on Magnetics, 48 (11), 3764-3767, (2012). https://dx.doi.org/10.1109/tmag.2012.2202885
11 Lee, D. W., Choi, J. Y., Cho, M. W., Lee, C. H., Cho, W. O., and Yun, H. C. Tribological characteristics in modified magneto-rheological fluid, Applied Mechanics and Materials, 110, 225-231, (2011). https://dx.doi.org/10.4028/www.scientific.net/amm.110116.225

12 Liu, X. H., Lu, H., Chen, Q. Q., Wang, D. D., and Zhen, X. J. Study on the preparation and properties of silicone oil-based magnetorheological fluids, Materials and Manufacturing Processes, 28 (6), 631-636, (2013). https://dx.doi.org/10.1080/10426914.2013.773017

13 Sutrisno, J., Fuchs, A., Sahin, H., and Gordaninejad, F. Surface coated iron particles via atom transfer radical polymerization for thermal-oxidatively stable high viscosity magnetorheological fluid, Journal of Applied Polymer Science, 128 (1), 470-480, (2013). https://dx.doi.org/10.1002/app.38199

14 Pramudya, I., Sutrisno, J., Fuchs, A., Kavlicoglu, B., Sahin, H., and Gordaninejad, F. Compressible magnetorheological fluids based on composite polyurethane microspheres, Macromolecular Materials and Engineering, 298 (8), 888895, (2013). https://dx.doi.org/10.1002/mame.201200156

15 Susan-Resiga, D. and Vks, L. Yield stress and flow behavior of concentrated ferrofluid-based magnetorheological fluids: The influence of composition, Rheologica Acta, 53 (8), 645-653, (2014). https://dx.doi.org/10.1007/s00397014-0785-z

16 Shah, K., Seong, M. S., Upadhyay, R. V., and Choi, S. B. A low sedimentation magnetorheological fluid based on platelike iron particles, and verification using a damper test, Smart Materials and Structures, 23 (2), 027001, (2014). https://dx.doi.org/10.1088/0964-1726/23/2/027001

17 Philips, R. W. Engineering applications of fluids with a variable yield stress. California: University of California, Berkeley, (1969). 